\title{
618.
}

\section{ON THE MECHANICAL DESCRIPTION OF A CARTESIAN.}

[From the Quarterly Journal of Pure and Applied Mathematics, vol. xIII. (1875), pp. 328-330.]

SuPPose that in two different curves the radius vectors $r, r^{\prime}$, which belong to the same angle $\theta$, are connected by the equation

$$
r^{2}+\left(M r^{\prime}+N+\frac{P}{r^{\prime}}\right) r+B=0
$$

then, taking one of the curves to be the circle

the other curve is

$$
M r^{\prime}+\frac{P}{r^{\prime}}=A \cos \theta
$$

$$
r^{2}+(A \cos \theta+N) r+B=0
$$

viz. this is a Cartesian. It perhaps would not be difficult to contrive a mechanical arrangement to connect the radius vectors in accordance with the foregoing equation; but the required result may be obtained equally well by means of a particular case of the relation in question; viz. taking this to be

$$
r^{2}+\left(-r^{\prime}+N\right) r+B=0
$$

then, taking the one curve to be the circle $r^{\prime}=-A \cos \theta$, the other curve is the Cartesian,

$$
r^{2}+(2 l \cos \theta+B) r+D=0, \text { that is, } r^{2}+(A \cos \theta+N) r+B=0 .
$$

The relation between the radius vectors may in this case be written

$$
r^{\prime}=N+r+\frac{B}{r}
$$

which can be constructed mechanically by a simple addition to the Peaucellier-cell, viz. if we joint on to $C$ (fig. 1, p. 536) a $\operatorname{rod} C D A$, having a slot, working on a pin at $A$, so that the rod is thereby kept always in the line $B A C$, then, making $B$ the 
fixed point and taking $B A=r$, we have $A C=\frac{m^{2}-l^{2}}{r}$, whence $B C=r+\frac{m^{2}-l^{2}}{r}$, or $D$ being a point at the distance $C D,=\alpha$, from the point $C$, and denoting $B D$ by $r^{\prime}$, we have $r^{\prime}=r+\frac{m^{2}-l^{2}}{r}+\alpha$, which is an equation of the required form; whence, if the point $D$ describe a circle passing through $B$, then the point $A$ will describe a Cartesian.

Fig. 1.

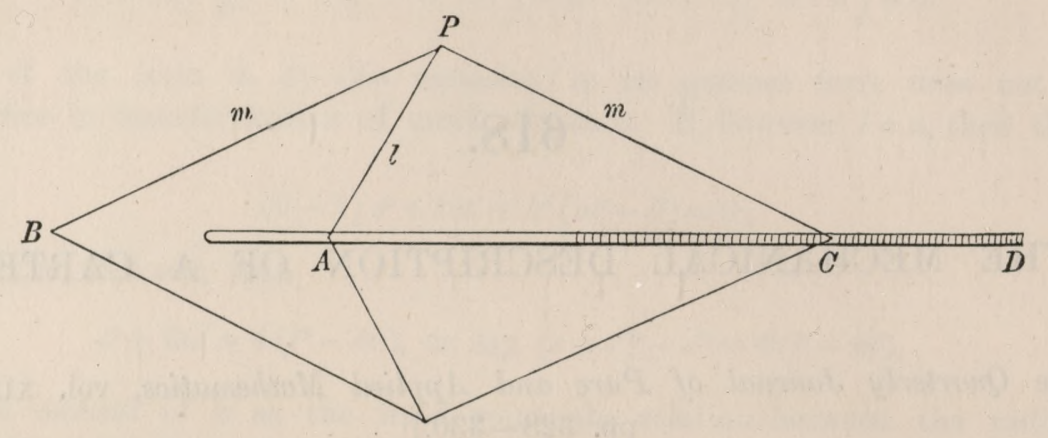

The equation of the Cartesian is $r^{2}+(A \cos \theta+N) r+B=0$, viz. this is $x^{2}+y^{2}+A x+B=-N \sqrt{ }\left(x^{2}+y^{2}\right)$, or writing $N^{2}=a$, it is

$$
\left(x^{2}+y^{2}+A x+B\right)^{2}=a x^{2}+a y^{2},
$$

which is the form considered in the preceding paper. It may be further observed in regard to it that, starting from the focal equation $r=l s+m$, where $r, s$ are the distances of a point $(x, y)$ of the Cartesian from any two of its three foci, this equation gives $r^{2}-l^{2} s^{2}+m^{2}=2 m r$, or writing $r^{2}=x^{2}+y^{2}, s^{2}=(x-\alpha)^{2}+y^{2}$, the function on the left-hand is of the form $\left(1-l^{2}\right)\left(x^{2}+y^{2}+A x+B\right)$, whence, assuming $\frac{2 m}{1-l^{2}}=\sqrt{ }(a)$, the equation becomes as above

$$
\left(x^{2}+y^{2}+A x+B\right)^{2}=a\left(x^{2}+y^{2}\right) .
$$

Taking the distance $r=\sqrt{ }\left(x^{2}+y^{2}\right)$ to be measured from a given focus, it is easy to see that, no matter which of the other two foci we associate with it, we obtain the same equation $\left(x^{2}+y^{2}+A x+B\right)^{2}=a\left(x^{2}+y^{2}\right)$; viz. starting with any one focus, we connect with it a determinate circle $x^{2}+y^{2}+A x+B=0$, and a determinate coefficient $a$, such that taking this focus as the origin, the equation of the curve is

$$
\left(x^{2}+y^{2}+A x+B\right)^{2}=a\left(x^{2}+y^{2}\right)
$$

but there are for the given curve three such forms of equation, according as the origin is taken at one or other of the three foci.

(Addition, Feb. 1875.) It is obviously the same thing, but $I$ find that it is mechanically more convenient to derive the Cartesian from the Limaçon $r^{\prime}=-N-A \cos \theta$, by the transformation $r^{\prime}=r+\frac{B}{r}$ : I have on this principle constructed an apparatus whereby the Cartesian is described on a rotating board by a pencil moving in a fixed line. 